\title{
Concentration of Lysozyme/Single-Walled Carbon Nanotube Dispersions
}

\author{
Daniel W. Horn and Virginia A. Davis* \\ Department of Chemical Engineering, Auburn University
}

KEYWORDS: Single-Walled Carbon Nanotubes, Lysozyme, Rheology, Viscoelasticity

*Corresponding author. davisva@auburn.edu

\begin{abstract}
The dispersion of single-walled carbon nanotubes (SWNT) in aqueous solutions of biological materials enables the production of bulk films and fibers that combine natural biological activity with SWNT's intrinsic mechanical, thermal, and electrical properties. In this work, we report the rheology and phase behavior of concentrated lysozyme (LSZ)/SWNT dispersions. Even at low concentration, the LSZ's globular structure causes a deviation from the rheological behavior expected of rigid rods such as SWNT. With increasing concentration, stabilized SWNT typically form lyotropic liquid crystalline phases. However, in this case, the LSZ results in depletion attraction and the formation of large dense SWNT aggregates surrounded by a LZ network. At intermediate concentrations, the microstructure and rheological properties are a complex function of the initial dispersion state, the absolute concentrations, and the LSZ to SWNT ratio. The rheological effects of concentrating mixtures comprised of aggregates, a range of bundle sizes, and individual SWNT were compared to the effects of concentrating supernatants comprised solely of individual SWNT and small bundles. In general, lysozyme concentration has the greatest impact on dispersion viscoelasticity. However, the inherent viscosity was a function
\end{abstract}


of SWNT concentration; data from both initial mixtures and supernatants spanning two orders of magnitude in concentration could be collapsed onto a single master curve. This work provides a foundation for exploring the behavior of other globular protein-SWNT dispersions.

\section{INTRODUCTION}

The unique electrical, mechanical, optical, and thermal properties of single-walled carbon nanotubes (SWNT) have been well documented.[1-3] There has also been a significant amount of research on a range of potential applications including polymer nanocomposites, [4-7] electrical thin films [8,9] and bio-inspired materials [10-15]. The antibacterial enzyme lysozyme (LSZ) has garnered significant attention as an intriguing SWNT dispersant. It has been considered as a model system for understanding fundamental nanotube-protein interactions [1421] as well as the effects of nanotubes on protein activity [18, 22-25], gelation, [26], crystallization [27], and separation [28, 29]. In addition, high strength, antimicrobial fibers [11, 30], and films [31] have been produced from LSZ/SWNT dispersions.

In spite of the many recent significant advances in understanding fundamental LSZSWNT interactions and the properties of bulk LSZ-SWNT films and fibers, there have been few studies aimed at probing the behavior of large volume LSZ-SWNT dispersions over a broad range of concentrations. Rheological characterization has emerged as a powerful tool for understanding both biological and SWNT dispersions because it is a bulk method that is effective regardless of dispersion composition or opacity. To date, rheological characterization of LSZ solutions has consisted primarily of interfacial rheology [32-34], rheology of LSZ gels [35-39] and liquid crystalline $[40,41]$ systems, or computational simulation of aqueous LSZ solutions [42]. Rheological characterization of SWNT dispersions has been used to improve understanding 
of many systems including SWNT in superacids [43], SWNT-polymer composites [5, 44-47], and aqueous SWNT-biomolecule dispersions [10, 48-50]. However, the majority of biomolecule dispersions have been lyotropic dispersions of SWNT and rod-like biomolecules such as deoxyribonucleic (DNA) and hyaluronic (HLA) acids. There has been limited phase behavior and rheological characterization of SWNT dispersions stabilized by roughly spherical biomolecules that can undergo depletion induced aggregation. A notable exception is Tardani et al 2014 which probed the incorporation SWNT into LSZ hydrogels [26].

In general, the phase behavior of any mixed colloidal system is determined by minimizing the total free energy of mixing. For entropically driven ordering, demixing is driven by differences in physical properties, such as shape, which can create bulk or microphase separation [51]. For rod-sphere systems, the physical and thermodynamic origin of this separation can be explained in terms of the depletion interaction between molecules which is entropically driven by the sum of electrostatic and steric repulsive forces, and van der Waals attractive forces [52-54]. When a rod and sphere approach each other, their individual depletion zones will overlap increasing the translational entropy of the system at the expense of the mixing entropy [55]. At low sphere concentration, the mixing entropy dominates and the mixture is miscible. However, with increasing sphere concentration, the mixing entropy decreases (due to the rise in translational entropy) and phase separation will occur [55-58]. Bulk phase separation, which is common in rod-sphere systems, occurs when the rods and spheres completely separate (in the absence of any interaction) forming a rod-rich and sphere-rich phase [59]. Another less common type of separation seen in rod-sphere systems is that of microphase separation. In microphase separation, the system starts demixing into regions that are either rod- or sphere-rich. However, unlike bulk separation, the liquid-like regions grow to a critical size at which point they can form kinetically 
stable three-dimensional equilibrium structures [51,59]. This last case is the one most applicable to LSZ/SWNT dispersions [11]. However, it is noted that LSZ/SWNT systems are expected to deviate from this theory for three primary reasons: 1. the aspect ratio of SWNT is significantly greater than rods typically used in the theoretical simulations [4], 2. LSZ is not perfectly spherical nor considered a hard sphere $[42,60,61]$, and 3. LSZ and SWNT interact with one another due to LSZ's polyampholitic character and $\pi-\pi$ interactions between SWNT and LSZ's tryptophan residue $[16,17,62]$.

We report the rheological properties of LSZ/SWNT dispersions as a function of relative and absolute concentrations and the initial LSZ/SWNT dispersion state. The results of this research show that the rheological properties are a complex function of dispersion state, and the absolute and relative concentrations. In general, the LSZ has a dominant contribution to viscoelasticity while the SWNT have a significant contribution to stead shear viscosity.

\section{MATERIALS \& METHODS}

Materials. The SWNT used in this research were Southwest Nanotechnology (Norman, OK) CG200-L100 SWNT made via the cobalt molybdenum catalyst (CoMoCat) process. The SWNT had a purity of $\geq 94 \%$ carbon as determined by thermogravimetric analysis. The average length and diameter were $\sim 860 \mathrm{~nm}$ and $\sim 1.36 \mathrm{~nm}$, respectively, as determined by atomic force microscopy. Dialyzed, lyophilized LSZ (L6876) from chicken egg white was purchased from Sigma Aldrich (St. Louis, MO) and used as delivered. The LSZ was determined to be $90 \%$ pure via spectroscopy with the impurities being buffer salts of sodium acetate and sodium chloride.

Sample Preparation. Dispersions of SWNT and LSZ were prepared in deionized DI water using tip sonication of aqueous SWNT-LSZ solutions. Sonication was performed using a Sonics 
(Newtown, CT) Vibra-Cell Model VC750 and a $13 \mathrm{~mm}$ diameter tip; the sample container was kept immersed in an ice bath to reduce sample heating. The sonication was 20 minutes in duration using pulses of 5 seconds on and 2 seconds off at wave amplitude of $20 \%$. The sonication waves temporarily overcome the $0.5 \mathrm{eV} / \mathrm{nm}$ van der Waals force of attraction between SWNT allowing LSZ to intercalate and stabilize SWNT as individuals and small bundles [3]. This has been described as bundle fraying followed by lysozyme attachment [63]. Since higher concentrations are desirable for processing into films and fibers, two sample preparation methods were investigated. First, the initial dispersions containing a mixture of individual SWNT and small aggregates were concentrated through evaporation. Second, centrifugation of LSZ/SWNT dispersions at 17,000xg for 3 hours was used to remove excess SWNT and LSZ, leaving a supernatant of predominantly individual SWNT. [11, 13, 15, 18]

Absorbance Spectroscopy. Absorption spectroscopy was used to create calibration curves for LSZ and LSZ/SWNT to determine supernatant and mixture concentrations. Approximately 300 $\mu \mathrm{L}$ dispersions of LSZ or LSZ/SWNT mixture or supernatant were then pipetted into $1 \mathrm{~mm}$ path length quartz UV-vis cell for scanning on a Cary (Santa Clara, CA) 3E UV-vis Spectrophotometer. Scans were performed at room temperature at wavelengths from $200 \mathrm{~nm}$ to $800 \mathrm{~nm}$ at a scan rate of $600 \mathrm{~nm} / \mathrm{s}$ and a resolution of $1 \mathrm{~nm}$.

Optical Microscopy. Optical microscopy was used to qualitatively determine the dispersion state and phase transition of LSZ/SWNT dispersions. Approximately $5 \mu \mathrm{L}$ of LSZ/SWNT mixture or supernatant dispersion were pipetted onto a glass slide. The pipette dispersion was the covered by a glass cover slip, and the edges were sealed to prevent evaporation. Images were captured on a Nikon (Melville, NY) Eclipse 80i optical microscope using with transmitted light with a 20x objective and 2x magnification in front of the color camera. 
Rheology. An Anton Paar (Ashland, VA) Physica MCR 301 with Peltier hood a $25 \mathrm{~mm}$ cone and plate geometry was used for rheological characterization. An amplitude sweep run at a constant frequency of $10 \mathrm{~s}^{-1}$ from 0.1 to $100 \%$ strain was used to determine the linear viscoelastic range and limit of linear viscoelasticity of the dispersions. A frequency sweep, run at a constant strain of $0.5 \%$ from an angular frequency of 0.1 to $100 \mathrm{~s}^{-1,}$ was used to probe the time-dependent oscillatory behavior of the dispersions. Transient tests run for $1000 \mathrm{~s}$ at a shear rate of $0.1 \mathrm{~s}^{-1}$ were used to find the time to reach a steady state viscosity. A steady-shear flow curve test run from a shear rate of 0.1 to $100 \mathrm{~s}^{-1}$ using a log-scale sampling time as determined from the transient testing was used to show how the viscosity of the dispersions varied with shear rate. All tests were run at $10^{\circ} \mathrm{C}$ to minimize evaporation. Each data set was collected over three separate loadings with three runs per loading for a total of nine runs per test.

Atomic Force Microscopy. A Pacific Nanotechnology (Berkley, CA) Nano-R SPM was used for performing atomic force microscopy (AFM) to determine the average length and diameter of the pristine SWNT used during research. Approximately $5 \mu \mathrm{L}$ of 0.25 wt.\% (0.15 vol.\%) LSZ 0.01 wt.\% (0.007 vol.\%) SWNT supernatant was pipetted onto a cleaned, molecularly flat silicon stub and dried in a vacuum oven at $30 \mathrm{mmHg}$ and $80^{\circ} \mathrm{C}$ for $24 \mathrm{~h}$. The AFM was operated in a static, non-contact mode. Each scan was of a $5 \mu \mathrm{m}^{2}$ area at a resolution of 256 and a scan speed of $0.5 \mathrm{~Hz}$.

Thermogravimetric Analysis. A TA Instruments (Waltham, MA) TGA Q50 was used to characterize SWNT and determine the relative SWNT and LSZ concentrations in concentrated samples. Mixture and supernatant dispersions were each prepared by removing water from the sample through non-heated, vacuum drying. After removal of the water, the remaining sample was placed in a sealed scintillation vial to prevent absorption of moisture. Approximately $20 \mathrm{mg}$ 
of sample was placed in a platinum sample pan to ensure adequate signal. Each dispersion was run in air and argon using the following test method: 1. Isothermal hold for $30 \mathrm{~min}$ at ambient temperature, 2. Temperature ramp to $120^{\circ} \mathrm{C}$ at $10^{\circ} \mathrm{C} / \mathrm{min}, 3$. Isothermal hold at $120^{\circ} \mathrm{C}$ for 30 min., 4. Temperature ramp to $800^{\circ} \mathrm{C}$ at $10^{\circ} \mathrm{C} / \mathrm{min}$., 5. Isothermal hold at $800^{\circ} \mathrm{C}$ for $45 \mathrm{~min}$.

\section{RESULTS \& DISCUSSION}

It has been recently established through independent experimental [11] and simulation [16, 17] research that the SWNT stabilization in aqueous LSZ is predominantly due to interactions with LSZ's tryptophan residue. However, the phase and rheological behavior of LSZ/SWNT dispersions is complex and not well understood. If the relative LSZ concentration is too low, there is insufficient SWNT stabilization and the SWNT reaggregate due to the strong van der Waals forces of attraction between. However, as shown in Figure 1, aggregation can also occur at higher LSZ:SWNT ratios. In the initial mixtures, the SWNT seemed to be generally well distributed with little to no evidence of large dense aggregates. For simplicity, this microstructure is referred to as "distributed." Increasing concentration via evaporation always resulted in an abrupt change to a "demixed" microstructure containing many large SWNT aggregates. This transition to a demixed state could also be detected rheologically by a both sudden increase in steady shear viscosity $\eta$ and decrease in the ratio of the viscous modulus to the elastic modulus $\tan (\delta)=G^{\prime} / G^{\prime}$.[11] For dispersions of rigid rods (including SWNT) aggregation typically results in the opposite behavior [64-66]; the reduction in interfacial surface area and aspect ratio that accompanies aggregation leads to a reduction in viscosity and increase in $\tan (\delta)[65,66]$. However, in the case of LSZ/SWNT aggregation is the result of depletion interactions between the spherical LSZ and cylindrical SWNT $[11,26]$. This results in an abrupt 
transition where the SWNT are forced together into aggregates surrounded by an elastic LSZ network; the loss of SWNT mixing entropy is compensated by an increase in the LSZ translational entropy. The microstructures shown in Figure 1 are not believed to represent thermodynamic equilibrium, but are the result of kinetically arrested microphase separation into amorphous aggregates. The attractive interaction induces a phase separation of the SWNT only when the LSZ concentration is sufficiently large. In fact, concentration of the much more dilute SWNT supernatants did not appear to result in depletion.

Since dispersions with large SWNT aggregates are not useful for producing advanced materials, characterizing the behavior of dispersions below the demixing concentration is of primary interest. By varying the LSZ:SWNT ratio it is possible to achieve a broader SWNT concentration range with a distributed microstructure. In this work, the initial mixtures had ratios of approximately $8.5,6.4,4.3$, and $2.1: 1 \mathrm{LSZ}$ :SWNT by volume; there was some variation in actual values due to experimental error. These mixtures were concentrated by evaporation on an orbital mixer to yield the concentrations shown in Figure 2. Equivalent mixtures were prepared and centrifuged to yield supernatants containing predominantly individual SWNT. These supernatants were also concentrated by evaporation on an orbital mixer to yield the concentrations shown in Figure 3. All concentrations are given as the concentration of LSZ / concentration of SWNT in vol. \%. It should be noted that for constant sonication conditions, the LSZ and SWNT concentrations in supernatants are strongly dependent on the relative initial concentrations, the type of SWNT, and the volume being sonicated. Volume has a particularly significant influence due to differences in energy density and mixing. For the same concentrations and sonication conditions, larger volumes result in a lower energy density which leads to less SWNT separation and stabilization, and the formation of fewer individual SWNT 
adducts. This in turn lowers the SWNT concentrations in the supernatants. [67, 68] In this work, $100 \mathrm{ml}$ mixtures were prepared in order to have sufficient fluid for rheological testing and/or films [12] and fibers [11]. The initial 8.5, 6.4, 4.3, and 2.1:1 LSZ:SWNT mixtures (by volume) resulted in supernatants with 58, 43, 28, and 14:1 LSZ:SWNT by volume. In other words, the LSZ:SWNT ratio in the supernatant was approximately seven times higher than that of the dispersion. For all but two of the dispersions, the supernatant LSZ concentration was approximately $75 \%$ of that in the mixture but the SWNT concentration was only $10 \%$ of that in the initial mixture.

Oscillatory rheology provided information on the effects of the relative and absolute concentrations on microstructure and viscoelasticity. For all samples, the linear viscoelastic region was $2.5 \%<\gamma_{c}<3.9 \%$, where $\gamma_{c}$ is the critical strain. Figure 2 shows the storage G' and loss G" moduli for the mixtures. In all the rheology figures, each symbol shape depicts an initial mixture or supernatant of fixed LSZ:SWNT ratio that was then concentrated; and the changes in symbol color depict increasing concentrations from that initial dispersion. For example, the red data indicated four initial mixtures with similar LSZ concentrations and different LSZ:SWNT ratios. All of these dispersions showed similar viscoelasticity. None of them exhibited terminal behavior; the low frequency slopes were $\sim 0.5$. Interestingly, the samples with the lowest SWNT concentrations had the higher values of G' and G'. This is likely due to a higher concentration of free lysozyme (unassociated with SWNT) in the lower concentration SWNT samples. The dispersions containing 0.50 to 0.57 vol.\% SWNT all had both higher values of G' and smaller low frequency slopes, even at low SWNT concentration. This highlights the significant contribution LSZ had on viscoelasticity. However, the absolute and relative SWNT concentrations also had an impact. The $0.63 / 0.15$ and $0.78 / 0.09 \mathrm{LSZ} / \mathrm{SWNT}$ samples had similar 
viscoelasticity, but the $0.73 / 0.11$ and $0.61 / 0.28$ samples had different moduli. In fact, for the $0.61 / 0.28,1.32 / 0.21$, and $1.58 / 0.18 \mathrm{G}^{\prime}$ was nearly independent of frequency suggesting that lysozyme contribute to gel formation while the SWNT concentration contributed to the magnitude of G'.

For the supernatant dispersions, the magnitudes of $G$ ' and G" were lowest for the 1.01/0.18 and 1.11/0.026 LSZ/SWNT dispersions. However, the 1.06/0.075 dispersion had one of the highest values of G'. All other concentrated supernatants showed rheological properties that were much less variant than the mixtures. This is likely due to all the supernatants initially consisting of well-dispersed, predominantly individual, SWNT-LSZ adducts as opposed to the highly variable blend of individuals, aggregates and bundles in the initial mixtures.

Figure 4 shows the steady shear viscosity of the mixture and supernatant dispersions as a function of shear rate. The dispersions behaved as power law fluids where $\eta=K \dot{\gamma}^{(\mathrm{n}-1)}$; the supernatants showed some evidence of a low shear plateau. For the entire concentration range studied, from 0.0050 vol. $\%$ SWNT to 0.4195 vol. $\%$ SWNT, $K$ varied from $0.25 \mathrm{~Pa} \mathrm{~s}^{2}$ to $10 \mathrm{~Pa} \mathrm{~s}^{2}$ and $n$ varied from 0.01 to 0.25 . For the supernatants and low viscosity dispersions, $0.16<\mathrm{n}<$ 0.20 while for the higher concentration mixtures $0.01<\mathrm{n}<0.03$. Unlike many SWNT dispersions which exhibit liquid crystalline behavior, the system did not display a yield stress, a maximum in the viscosity versus concentration curve or other characteristics of lyotropic liquid crystalline behavior. This supports that the presence and network formation of the globular LSZ protein suppressed SWNT's lyotropic phase behavior [10, 43, 69, 70]. The results shown in Figure 4 suggest that the SWNT concentration had the greatest effect on viscosity. At a shear rate of $0.01 \mathrm{~s}^{-1}$, even the lowest concentration sample had a viscosity four orders of magnitude greater than that of water. The increase from 0.05 to 0.42 vol. \% SWNT results in another two 
order of magnitude increase to nearly $1000 \mathrm{~Pa}$ s. This behavior is consistent with expectations for high aspect ratio rods. The rotary diffusivity $\mathrm{D}_{\mathrm{r} 0}$ of the $0.295 / 0.005 \mathrm{LSZ} / \mathrm{SWNT}$ supernatant was calculated as $D_{r 0}=\frac{3 k_{B} T\left(\ln \left(\frac{L}{d}\right)-0.8\right)}{\pi \eta_{S} L^{3}}=17.16 \mathrm{~s}^{-1}$ which results in a Peclet number $P e \equiv \frac{\dot{\gamma}}{D_{r}}=$ 0.0005 which suggests that Brownian motion is dominant.

However, the LSZ concentration and LSZ:SWNT also affected the viscosity. For approximately the same SWNT concentrations, higher LSZ concentration resulted in higher viscosity. For example, the low shear viscosity of the $0.39 / 0.041$ sample was 17 Pa s compared to $31 \mathrm{~Pa}$ s for $0.55 / 0.09$ and 40 for the $0.78 / 0.09$ mixtures. For the supernatants, the two highest LSZ concentrations had the lowest viscosity; both $1.02 / 0.018$ and $1.11 / 0.026$ had low shear viscosities of approximately $2 \mathrm{~Pa}$ s. However, for the $0.836 / 0.030$ dispersion $\eta=12 \mathrm{~Pa}$; ; this suggests that the higher LSZ concentration samples may have undergone some demixing. For the supernatant samples, the LSZ and SWNT have more of a combined contribution to viscosity. All the other samples had a low shear viscosities on the order of $10 \mathrm{~Pa} \mathrm{~s}$ in spite of a SWNT concentration ranging from 0.005 to 0.042 .

The relative contribution of the SWNT to the dispersions' viscosities was quantified in terms of the reduced $\eta_{\mathrm{R}}$, intrinsic $[\eta]$ and inherent $\eta_{\mathrm{inh}}$ viscosities:

$$
\begin{array}{r}
\eta_{R}=\frac{\eta-\eta_{S}}{\eta_{S} \phi_{S W N T}} \\
{[\eta]=\lim _{\phi \rightarrow 0} \eta_{R}} \\
\eta_{\text {inh }}=\frac{\ln \left(\eta / \eta_{s}\right)}{\phi_{S W N T}}
\end{array}
$$

where $\eta$ is the dispersion viscosity, $\eta_{\mathrm{s}}$ is the viscosity of a solution with the same lysozyme concentration as the dispersion, and $\phi_{\mathrm{SWNT}}$ is the SWNT volume fraction. According to 
Kirkwood and Plock (1956), for Brownian rods, a plot of $\eta_{R}$ versus Weissenberg number Wi should have a slope of -0.5 for $\mathrm{Wi}>1$ [71]. This behavior was exhibited for dilute dispersions of SWNT in superacids [43]. However, in the case of LSZ/SWNT, the slope is $\sim-0.8$; this further underscores that the presence of the LSZ results in a deviation from rigid-rod behavior. In spite of this deviation, fitting a linear trendline through the low shear dilute reduced viscosity solution data and extrapolating to zero concentration resulted in an intrinsic viscosity $[\eta]=7290$. This value is within the range of $7235<[\eta]>9130$ which has reported for HiPCo SWNT in superacids and surfactants $[43,72]$. Interestingly, as shown in Figure 5 plotting $\eta_{\text {inh }}$ versus concentration for all dispersions results in a master curve in spite of the large concentration range, different LSZ:SWNT ratios, and the fact some dispersions were concentrated from supernatants of individual SWNT and some dispersions were concentrated from initial mixtures that contained both individual and bundled SWNT. This highlights that the fundamental interactions governing the system are scalable and independent of the nature of the initial dispersion.

\section{CONCLUSIONS}

In the last few years, there have been significant advances in understanding the molecular interactions between LSZ and SWNT and in producing antimicrobial LSZ/SWNT materials. Further advancing the field requires understanding the microstructure and bulk rheological properties of LSZ/SWNT dispersions. The results of this work highlight that although LSZ is a powerful SWNT dispersant, careful consideration needs to be given to the absolute and relative concentrations when preparing dispersions. Even in the dilute regime, the presence of the globular lysozyme results in deviation from the rheological behavior expected for Brownian 
rods. In spite of this, the measured intrinsic viscosities are within literature values for individual SWNT. At high concentration, free lysozyme results in depletion attraction between SWNT and the formation of large, dense aggregates. The rheological properties of dispersions concentrated form both the mixtures and supernatants, were a complex function of absolute and relative concentrations. The steady shear and oscillatory rheological properties of the supernatants were more affected by absolute and relative concentrations than those of the supernatant. This is attributed to all supernatants having a similar initial microstructure of predominantly individual SWNT while the mixtures had a range of floc, bundle and aggregate densities and sizes. In general, the lysozyme concentration had the greatest impact on viscoelasticity while the SWNT had the greatest impact on steady shear viscosity. In spite of system complexity, the inherent viscosities of all dispersions collapsed onto a single master curve showing power law dependence of inherent viscosity on SWNT concentration.

\section{ACKNOWLEDGMENTS}

This research was supported by National Science Foundation Grant (CMMI-0846629). The authors acknowledge Philippe Poulin for useful discussions and contributions to related research. The assistance of Geyou Ao, Joyanta Goswami, and Ange Nyankima is also acknowledged. 


\section{REFERENCES}

[1] M. Dresselhaus, G. Dresselhaus, R. Saito, Physics of carbon nanotubes, Carbon, 33 (1995) 883-891.

[2] M.S. Dresselhaus, G. Dresselhaus, P. Eklund, D.E.H. Jones, Science of fullerenes and carbon nanotubes, Academic Press New York1996.

[3] A. Thess, R. Lee, P. Nikolaev, H. Dai, P. Petit, J. Robert, C. Xu, Y.H. Lee, S.G. Kim, A.G. Rinzler, Crystalline ropes of metallic carbon nanotubes, Science, 273 (1996) 483.

[4] J. Vavro, M.C. Llaguno, J.E. Fischer, S. Ramesh, R.K. Saini, L.M. Ericson, V.A. Davis, R.H. Hauge, M. Pasquali, R.E. Smalley, Thermoelectric power of p-doped single wall carbon nanotubes and the role of phonon drag, Phys. Rev. Lett., 90 (2003) 065503.

[5] F. Du, R.C. Scogna, W. Zhou, S. Brand, J.E. Fischer, K.I. Winey, Nanotube networks in polymer nanocomposites: rheology and electrical conductivity, Macromolecules, 37 (2004) 9048-9055.

[6] P.M. Ajayan, L.S. Schadler, C. Giannaris, A. Rubio, Single-walled carbon nanotube-polymer composites: strength and weakness, Advanced Materials, 12 (2000) 750-753.

[7] M. Moniruzzaman, K.I. Winey, Polymer nanocomposites containing carbon nanotubes, Macromolecules, 39 (2006) 5194-5205.

[8] P.G. Collins, P. Avouris, Nanotubes for electronics, Scientific American, 283 (2000) 62-69.

[9] P. Corio, A. Jorio, N. Demir, M. Dresselhaus, Spectro-electrochemical studies of single wall carbon nanotubes films, Chemical physics letters, 392 (2004) 396-402.

[10] G. Ao, D. Nepal, M. Aono, V.A. Davis, Cholesteric and Nematic Liquid Crystalline Phase Behavior of Double-Stranded DNA Stabilized Single-Walled Carbon Nanotube Dispersions, ACS Nano, 5 (2011) 1450-1458. 
[11] D.W. Horn, G. Ao, M. Maugey, C. Zakri, P. Poulin, V.A. Davis, Dispersion State and Fiber Toughness: Antibacterial Lysozyme-Single Walled Carbon Nanotubes, Adv. Funct. Mater., (2013) 6082-6090

[12] A.G. Nyankima, D.W. Horn, V.A. Davis, Free-Standing Films from Aqueous Dispersions of Lysozyme, Single-Walled Carbon Nanotubes, and Polyvinyl Alcohol, ACS Macro Letters, 3 (2013) 77-79.

[13] D. Nepal, S. Balasubramanian, A.L. Simonian, V.A. Davis, Strong Antimicrobial Coatings: Single-Walled Carbon Nanotubes Armored with Biopolymers, Nano Lett., 8 (2008) 1896-1901.

[14] D. Nepal, K.E. Geckeler, pH-Sensitive Dispersion and Debundling of Single-Walled Carbon Nanotubes: Lysozyme as a Tool, Small, 2 (2006) 406-412.

[15] D. Nepal, K.E. Geckeler, Proteins and carbon nanotubes: Close encounter in water, Small, 3 (2007) 1259-1265.

[16] M. Calvaresi, F. Zerbetto, The Devil and Holy Water: Protein and Carbon Nanotube Hybrids, Acc. Chem. Res., 46 (2013) 2454-2463.

[17] M. Calvaresi, S. Hoefinger, F. Zerbetto, Probing the Structure of Lysozyme-CarbonNanotube Hybrids with Molecular Dynamics, Chemistry - A European Journal, 18 (2012) 43084313.

[18] D.W. Horn, K. Tracy, C.J. Easley, V.A. Davis, Lysozyme Dispersed Single-Walled Carbon Nanotubes: Interaction and Activity, The Journal of Physical Chemistry C, 116 (2012) 1034110348.

[19] F. Bomboi, A. Bonincontro, C. La Mesa, F. Tardani, Interactions between single-walled carbon nanotubes and lysozyme, J. Colloid Interface Sci., 355 (2011) 342-347. 
[20] H.Y. Nie, H.F. Wang, A.N. Cao, Z.J. Shi, S.T. Yang, Y.A. Yuan, Y.F. Liu, Diameterselective dispersion of double-walled carbon nanotubes by lysozyme, Nanoscale, 3 (2011) 970973.

[21] G. Raffaini, F. Ganazzoli, Protein adsorption on biomaterial and nanomaterial surfaces: a molecular modeling approach to study non-covalent interactions, J. Appl. Biomater. Biomech., 8 (2010) 135-145.

[22] D. Merli, M. Ugonino, A. Profumo, M. Fagnoni, E. Quartarone, P. Mustarelli, L. Visai, M.S. Grandi, P. Galinetto, P. Canton, Increasing the Antibacterial Effect of Lysozyme by Immobilization on Multi-Walled Carbon Nanotubes, Journal of Nanoscience and Nanotechnology, 11 (2011) 3100-3106.

[23] Z. Borzooeian, A. Safavi, M.H. Sheikhi, M. Aminlari, M.M. Doroodmand, Preparation and investigation on properties of lysozyme chemically bonded to single-walled carbon nanotubes, J. Exp. Nanosci., 5 (2010) 536-547.

[24] L.M. Xie, S.G. Chou, A. Pande, J. Pande, J. Zhang, M.S. Dresselhaus, J. Kong, Z.F. Liu, Single-Walled Carbon Nanotubes Probing the Denaturation of Lysozyme, Journal of Physical Chemistry C, 114 (2010) 7717-7720.

[25] P. Asuri, S.S. Bale, R.C. Pangule, D.A. Shah, R.S. Kane, J.S. Dordick, Structure, function, and stability of enzymes covalently attached to single-walled carbon nanotubes, Langmuir, 23 (2007) 12318-12321.

[26] F. Tardani, C. La Mesa, Effects of single-walled carbon nanotubes on lysozyme gelation, Colloids Surf. B. Biointerfaces, 121 (2014) 165-170. 
[27] P. Asanithi, E. Saridakis, L. Govada, I. Jurewicz, E.W. Brunner, R. Ponnusamy, J.A.S. Cleaver, A.B. Dalton, N.E. Chayen, R.P. Sear, Carbon-Nanotube-Based Materials for Protein Crystallization, ACS Applied Materials \& Interfaces, 1 (2009) 1203-1210.

[28] Z. Du, N. Mang, J.H. Wang, On-line isolation of lysozyme from egg-white using multiwalled carbon nanotubes, Chem. J. Chin. Univ.-Chin., 29 (2008) 902-905.

[29] Z. Du, S. Zhang, C. Zhou, M. Liu, G. Li, Dynamic layer-by-layer self-assembly of multiwalled carbon nanotubes on quartz wool for on-line separation of lysozyme in egg white, Talanta, 94 (2012) 104-110.

[30] D. Nepal, M.L. Minus, S. Kumar, Lysozyme Coated DNA and DNA/SWNT Fibers by Solution Spinning, Macromol. Biosci., 11 (2011) 875-881.

[31] A.G. Nyankima, D.W. Horn, V.A. Davis, Free-Standing Films from Aqueous Dispersions of Lysozyme, Single-Walled Carbon Nanotubes, and Polyvinyl Alcohol, ACS Macro Letters, 3 (2013) 77-79.

[32] S.Y. Nishimura, G.M. Magana, H.A. Ketelson, G.G. Fuller, Effect of lysozyme adsorption on the interfacial rheology of DPPC and cholesteryl myristate films, Langmuir, 24 (2008) 1172811733.

[33] E.M. Freer, K.S. Yim, G.G. Fuller, C.J. Radke, Interfacial rheology of globular and flexible proteins at the hexadecane/water interface: comparison of shear and dilatation deformation, The Journal of Physical Chemistry B, 108 (2004) 3835-3844.

[34] S.A. Roberts, I.W. Kellaway, K.M.G. Taylor, B. Warburton, K. Peters, Combined surface pressure-interfacial shear rheology studies of the interaction of proteins with spread phospholipid monolayers at the air-water interface, International journal of pharmaceutics, 300 (2005) 48-55. 
[35] M.A. da Silva, E.P.G. Areas, Solvent-induced lysozyme gels: Rheology, fractal analysis, and sol-gel kinetics, Journal of colloid and interface science, 289 (2005) 394-401.

[36] M.A. da Silva, I.A. Farhat, E.P.G. Arêas, J.R. Mitchell, Solvent-induced lysozyme gels: Effects of system composition and temperature on structural and dynamic characteristics, Biopolymers, 83 (2006) 443-454.

[37] M.A. da Silva, R. Itri, E.P.G. Aręas, Lysozyme viscoelastic matrices in tetramethylurea/water media: a small angle X-ray scattering study, Biophysical chemistry, 99 (2002) 169-179.

[38] T. Kamiyama, M. Morita, T. Kimura, Rheological study of lysozyme in dimethyl sulfoxide+ water solution at $298.15 \mathrm{~K}$, Journal of Chemical \& Engineering Data, 49 (2004) 1350-1353.

[39] T. Knubovets, J.J. Osterhout, P.J. Connolly, A.M. Klibanov, Structure, thermostability, and conformational flexibility of hen egg-white lysozyme dissolved in glycerol, Proceedings of the National Academy of Sciences, 96 (1999) 1262.

[40] T. Mishraki, D. Libster, A. Aserin, N. Garti, Lysozyme entrapped within reverse hexagonal mesophases: Physical properties and structural behavior, Colloids and Surfaces B: Biointerfaces, 75 (2010) 47-56.

[41] T. Mishraki, D. Libster, A. Aserin, N. Garti, Temperature-dependent behavior of lysozyme within the reverse hexagonal mesophases (HII), Colloids and Surfaces B: Biointerfaces, 75 (2010) 391-397.

[42] F. Cardinaux, E. Zaccarelli, A. Stradner, S. Bucciarelli, B. Farago, S.U. Egelhaaf, F. Sciortino, P. Schurtenberger, Cluster-Driven Dynamical Arrest in Concentrated Lysozyme Solutions, The Journal of Physical Chemistry B, (2011). 
[43] V.A. Davis, L.M. Ericson, A.N. Parra-Vasquez, H. Fan, Y. Wang, V. Prieto, J.A. Longoria, S. Ramesh, R. Saini, C. Kittrell, W.E. Billups, W.W. Adams, R.H. Hauge, R.E. Smalley, M. Pasquali, Phase Behavior and Rheology of SWNTs in Superacids, Macromolecules, 37 (2004) 154-160.

[44] E.E. Ureña-Benavides, M.J. Kayatin, V.A. Davis, Dispersion and Rheology of Multiwalled Carbon Nanotubes in Unsaturated Polyester Resin, Macromolecules, 46 (2013) 1642-1650.

[45] E.K. Hobbie, D.J. Fry, Rheology of concentrated carbon nanotube suspensions, J. Chem. Phys., 126 (2007).

[46] T. Chatterjee, K. Yurekli, V.G. Hadjiev, R. Krishnamoorti, Single-Walled Carbon Nanotube Dispersions in Poly(ethylene oxide), Adv. Funct. Mater., 15 (2005) 1832-1838.

[47] M.J. Kayatin, V.A. Davis, Viscoelasticity and Shear Stability of Single-Walled Carbon Nanotube/Unsaturated Polyester Resin Dispersions, Macromolecules, 42 (2009) 6624-6632.

[48] S.E. Moulton, M. Maugey, P. Poulin, G.G. Wallace, Liquid Crystal Behavior of SingleWalled Carbon Nanotubes Dispersed in Biological Hyaluronic Acid Solutions, J. Am. Chem. Soc., 129 (2007) 9452-9457.

[49] V.A. Davis, Liquid crystalline assembly of nanocylinders, J. Mater. Res., 26 (2011) 140 153

[50] E. Cheng, Y. Li, Z. Yang, Z. Deng, D. Liu, DNA-SWNT hybrid hydrogel, Chemical Communications, (2011).

[51] M. Adams, Z. Dogic, S.L. Keller, S. Fraden, Entropically driven microphase transitions in mixtures of colloidal rods and spheres, nature, 393 (1998) 349-352.

[52] W. Poon, Crystallization of globular proteins, Physical Review E, 55 (1997) 3762-3764. 
[53] S. Asakura, F. Oosawa, Interaction between particles suspended in solutions of macromolecules, Journal of Polymer Science, 33 (1958) 183-192.

[54] T. Schilling, S. Jungblut, M.A. Miller, Depletion-induced percolation in networks of nanorods, Physical Review Letters, 98 (2007) 108303.

[55] G. Vliegenthart, H. Lekkerkerker, Phase behavior of colloidal rod-sphere mixtures, The Journal of Chemical Physics, 111 (1999) 4153.

[56] N. Urakami, M. Imai, Dependence on sphere size of the phase behavior of mixtures of rods and spheres, The Journal of Chemical Physics, 119 (2003) 2463.

[57] A. Cuetos, B. Martínez-Haya, S. Lago, L.F. Rull, Use of Parsons-Lee and Onsager theories to predict nematic and demixing behavior in binary mixtures of hard rods and hard spheres, Physical Review E, 75 (2007) 061701.

[58] Z. Dogic, S. Fraden, Phase Behavior of Rod-Like Viruses and Virus-Sphere Mixtures, Soft Matter, (2006) 1-86.

[59] Z. Dogic, D. Frenkel, S. Fraden, Enhanced stability of layered phases in parallel hard spherocylinders due to addition of hard spheres, Physical Review E, 62 (2000) 3925-3933.

[60] F. Ahmad, C. Contaxis, C.C. Bigelow, Free energy changes in lysozyme denaturation, Journal of Biological Chemistry, 258 (1983) 7960.

[61] F. Tanaka, L.S. Forster, P.K. Pal, J.A. Rupley, The circular dichroism of lysozyme, Journal of Biological Chemistry, 250 (1975) 6977.

[62] D. Horn, K. Tracy, C.J. Easley, V. Davis, Lysozyme Dispersed Single-Walled Carbon Nanotubes: Interaction and Activity, The Journal of Physical Chemistry C, (2012). 
[63] K. Matsuura, T. Saito, T. Okazaki, S. Ohshima, M. Yumura, S. Iijima, Selectivity of watersoluble proteins in single-walled carbon nanotube dispersions, Chem. Phys. Lett., 429 (2006) 497-502.

[64] M. Doi, S.F. Edwards, The theory of polymer dynamics, Oxford University Press, USA1988.

[65] C.W. Macosko, R.G. Larson, Rheology: principles, measurements, and applications, VCH New York1994.

[66] R.G. Larson, The structure and rheology of complex fluids, New York: Oxford, (2001).

[67] Q. Cheng, S. Debnath, E. Gregan, H.J. Byrne, Ultrasound-assisted SWNTs dispersion: effects of sonication parameters and solvent properties, The Journal of Physical Chemistry C, 114 (2010) 8821-8827.

[68] B. Ruan, A.M. Jacobi, Ultrasonication effects on thermal and rheological properties of carbon nanotube suspensions, Nanoscale research letters, 7 (2012) 1-14.

[69] V.A. Davis, A.N.G. Parra-Vasquez, M.J. Green, P.K. Rai, N. Behabtu, V. Prieto, R.D. Booker, J. Schmidt, E. Kesselman, W. Zhou, H. Fan, W.W. Adams, R.H. Hauge, J.E. Fischer, Y. Cohen, Y. Talmon, R.E. Smalley, M. Pasquali, True solutions of single-walled carbon nanotubes for assembly into macroscopic materials, Nature Nanotechnology, 4 (2009) 830-834.

[70] M.J. Green, A.N.G. Parra-Vasquez, N. Behabtu, M. Pasquali, Modeling the phase behavior of polydisperse rigid rods with attractive interactions with applications to single-walled carbon nanotubes in superacids, J. Chem. Phys., 131 (2009) 041401.

[71] J.G. Kirkwood, R.J. Plock, Non-Newtonian Viscoelastic Properties of Rod-Like Molecules in Solution, J. Chem. Phys., 24 (1956) 665-669. 
[72] A.N.G. Parra-Vasquez, I. Stepanek, V.A. Davis, V.C. Moore, E.H. Haroz, J. Shaver, R.H. Hauge, R.E. Smalley, M. Pasquali, Simple length determination of single-walled carbon nanotubes by viscosity measurements in dilute suspensions, Macromolecules, 40 (2007) 40434047. 


\section{FIGURES}

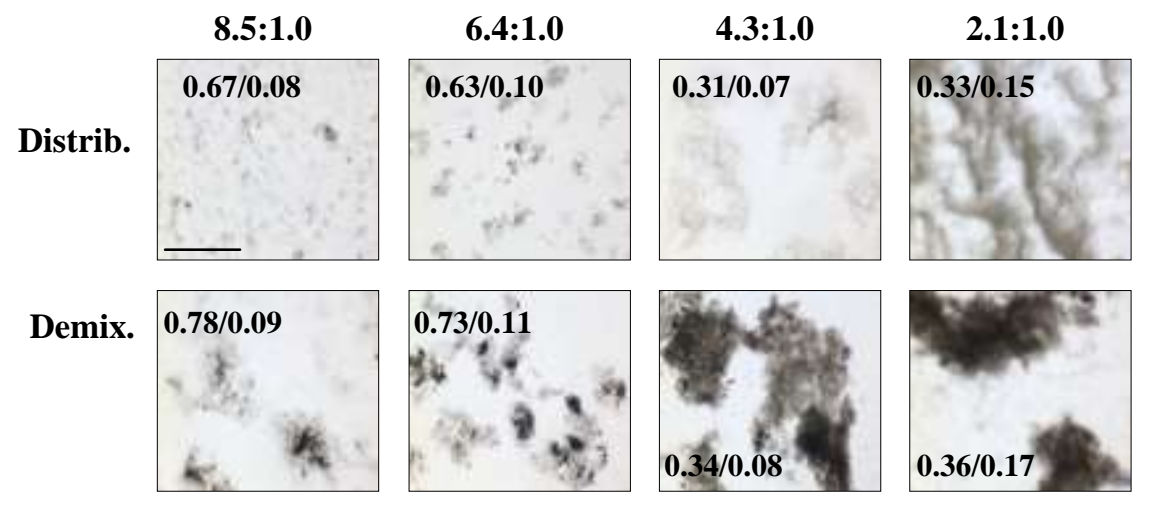

Figure 1. Optical microscopy images of dispersions and corresponding concentrations. Scale bar is $100 \mu \mathrm{m}$.

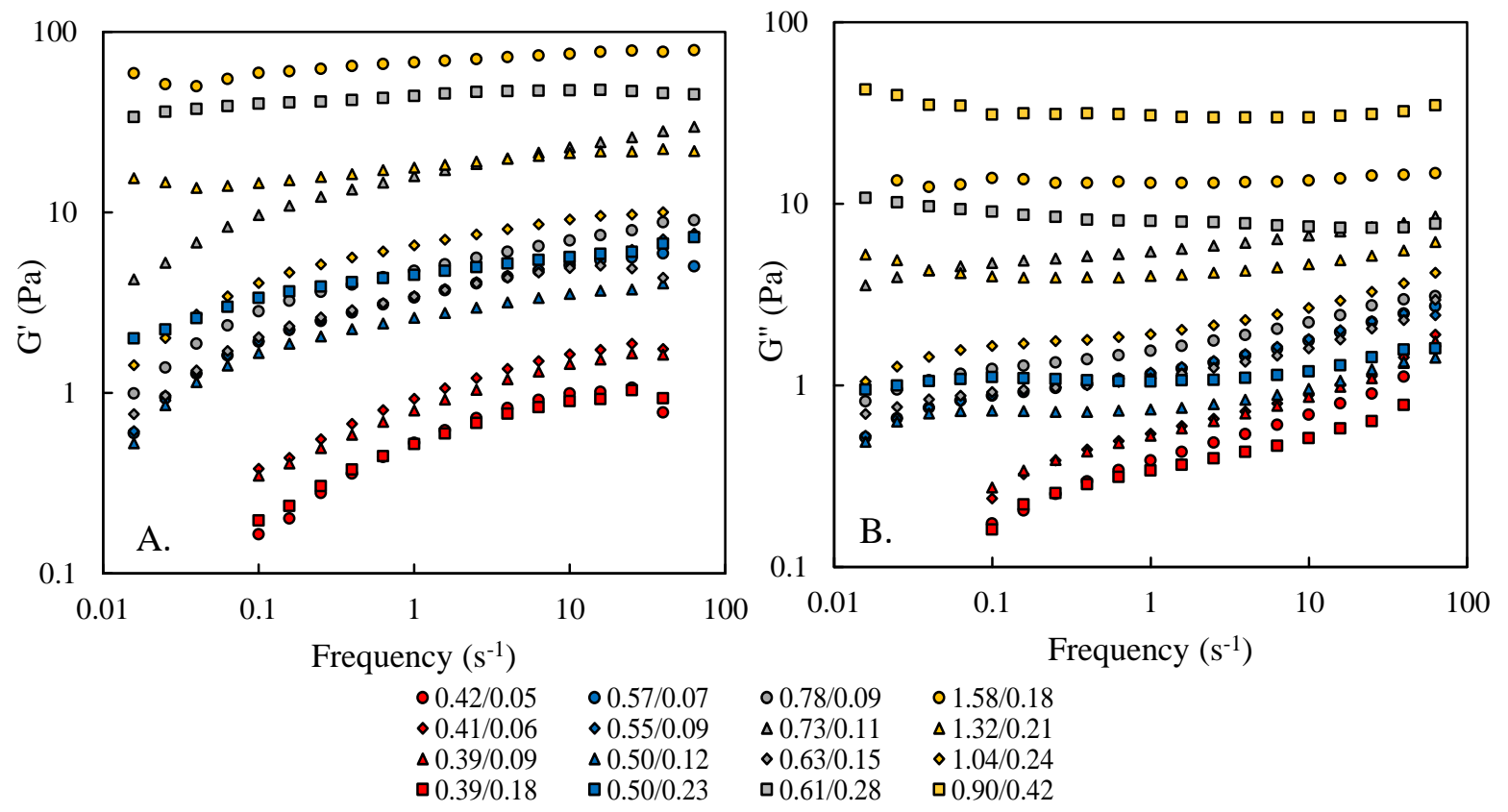

Figure 2. Linear viscoelastic oscillatory response showing A.) storage modulus $G$ ' and b.) loss modulus $G^{\prime}$ ' as a function of angular frequency $\omega$ for mixtures concentrated from four initial dispersions. 

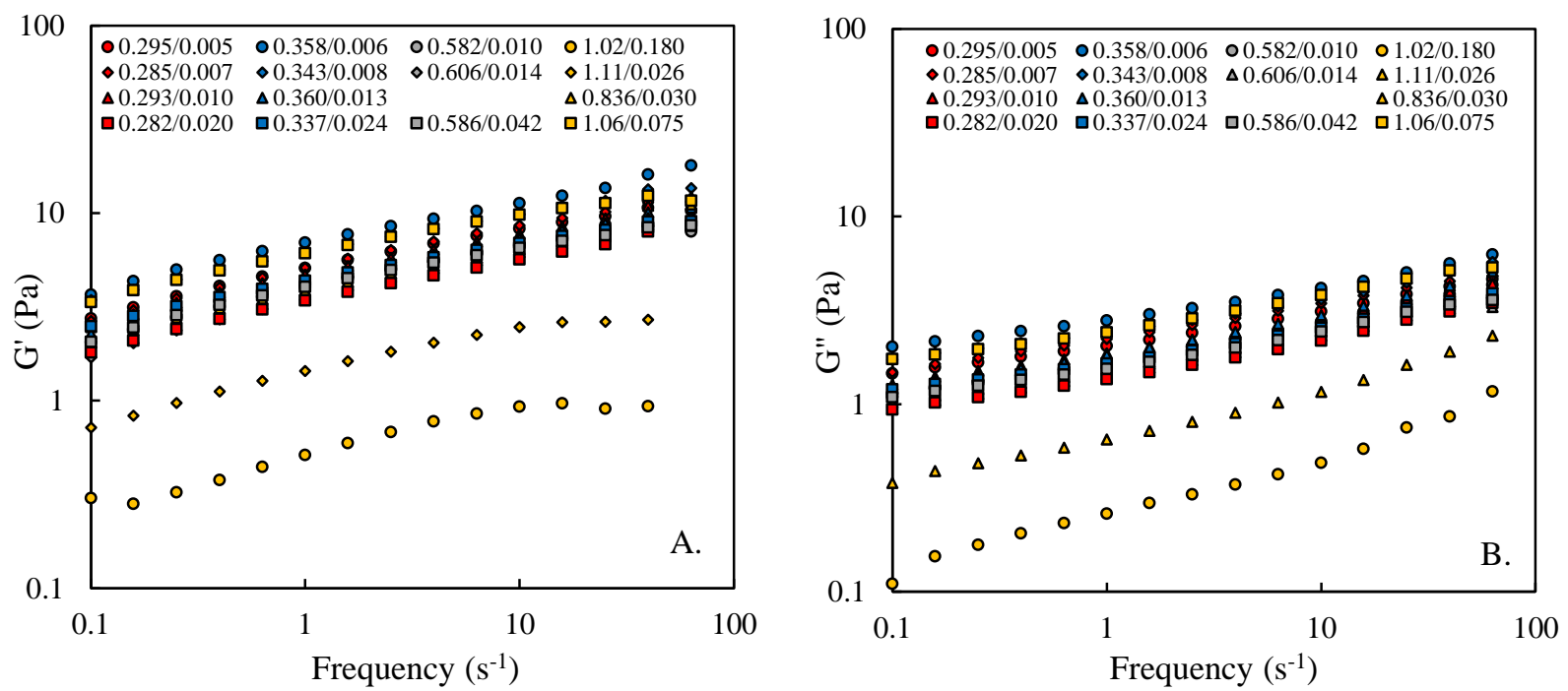

Figure 3. Linear viscoelastic oscillatory response showing A.) storage modulus $G^{\prime}$ and B.) loss modulus $G$ ', as a function of angular frequency $\omega$ for supernatants obtained from the initial mixtures and concentrated by evaporation.
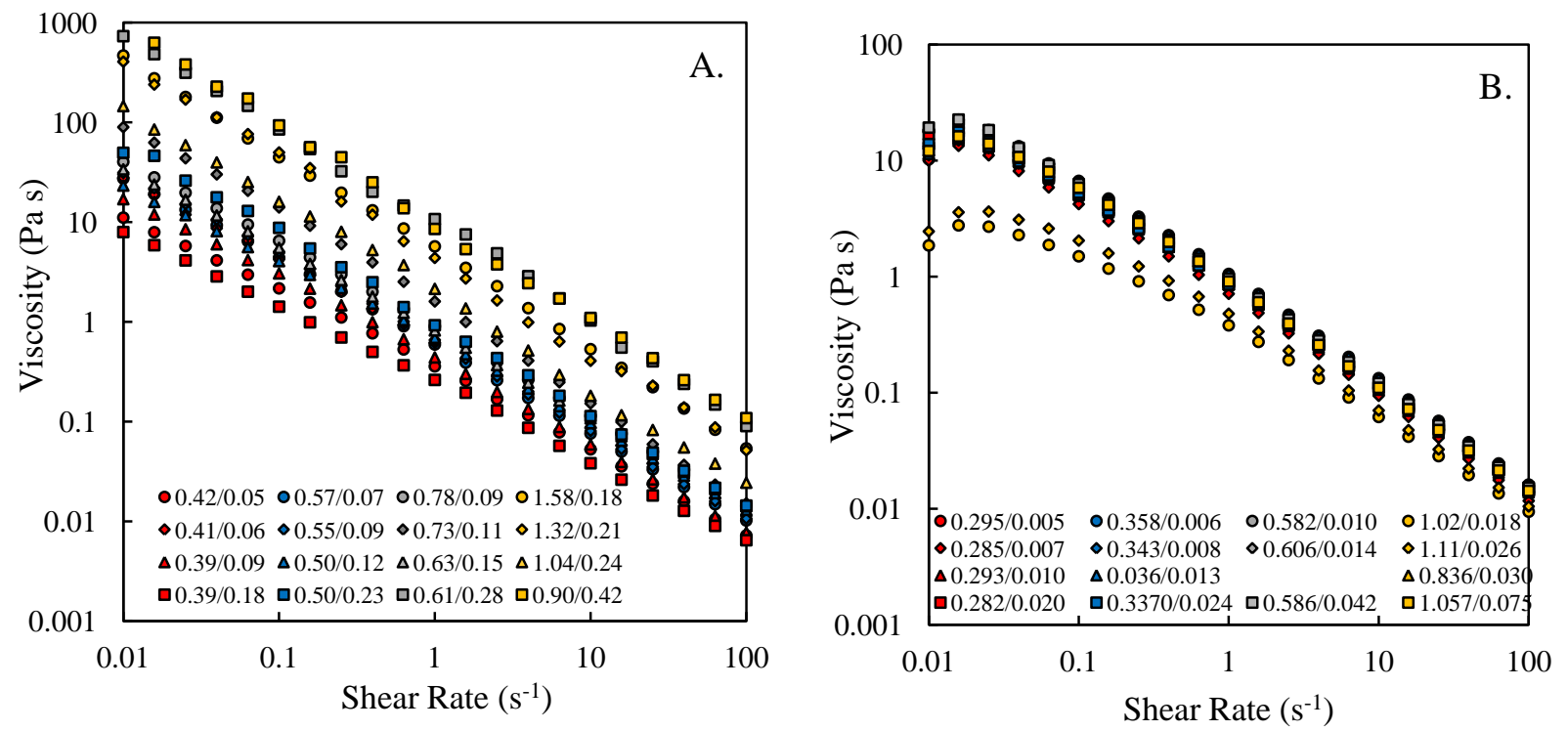

Figure 4. Viscosity versus shear rate A. mixtures, B. supernatants. Each shape represents a single dispersion that was concentrated. Error for each concentration is less than $+/-5 \%$. 


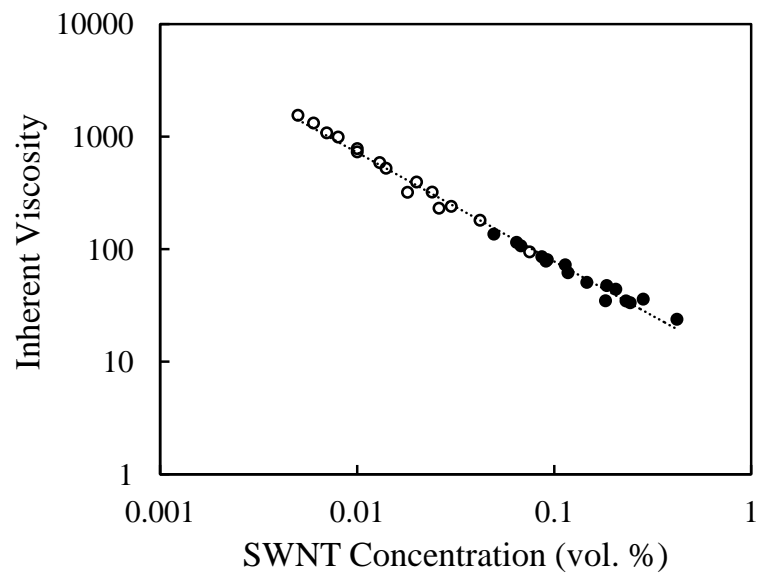

Figure 5. a.) Inherent viscosity versus SWNT concentration at a shear rate of $0.1 \mathrm{~s}^{-1}$ showing power law fit $\eta_{\text {Inherent }}=8.39 \phi_{S W N T}^{-0.968} \mathrm{R}^{2}=0.99$. 


\section{Graphical Abstract}

Concentration of Lysozyme/Single Walled Carbon Nanotube Dispersions

Daniel W. Horn and Virginia A. Davis*

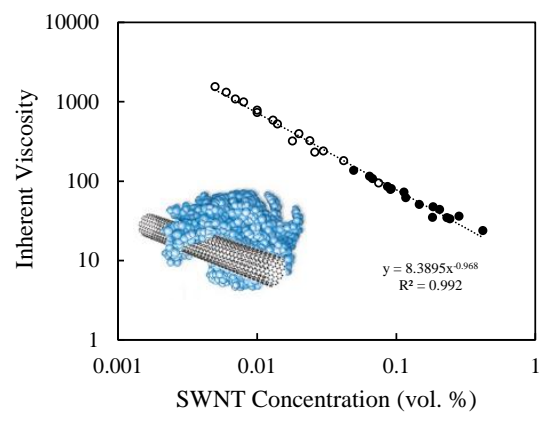

Superposition of lysozyme / SWNT dispersions' inherent viscosities. 